\title{
Abundances of [WC] Central Stars of PN and the Double Dust Chemistry Problem
}

\author{
Orsola De Marco \\ American Museum of Natural History, New York, NY 10024, USA
}

\begin{abstract}
The chemical abundances of Wolf-Rayet ([WC]) central stars are reviewed. Although the evolutionary connection between cooler (late) and hotter (early) [WC] central stars and PG1159 central stars is now on solid ground, several issues still remain. In particular, hydrogen and oxygen abundances, that could provide a way of discriminating between different evolutionary channels, are affected by large uncertainties. Following the ground-breaking discovery that cool [WC] central stars harbor hot carbon- as well as hot oxygen-rich dust, current theories of their origin have been put in serious doubt. Observations of three cool [WC] central stars are presented here which, while confirming the deficiency of our theoretical understanding, hint at the possibility of alternative scenarios, such as dust-storing disks.
\end{abstract}

\section{Introduction}

Wolf-Rayet central stars (CS; also referred to as [WC] CS, to differentiate them from massive Wolf-Rayet stars; van der Hucht et al. 1981) of planetary nebula $(\mathrm{PN})$ are H-deficient emission line stars. They comprise $\sim 7 \%$ of the entire CS population (Tylenda 1996). Several [WC] CSs have been analysed via non-Local Thermodynamic Equilibrium (LTE) modeling codes (e.g. Hillier \& Miller 1998), to determine their physical parameters and abundances (Leuenhagen, Hamann \& Jeffery 1996; Leuenhagen \& Hamann 1998; De Marco \& Crowther 1998,1999; De Marco et al. 2001; Koesterke \& Hamann 1997a,1997b; Table 1). These efforts showed the [WCL] ( $L$, for "Late") CSs to be precursors of the [WCE] ( $E$, for "Early") CSs, which in turn are precursor of the PG1159 CSs (Werner 2001). Together, these three groups comprise $\sim 9 \%$ of the CS population ${ }^{7}$.

Abundance studies have also revealed that the weak emission line CSs (WELS; 38 objects; Tylenda, Acker \& Stenholm 1993) which have been considered close associates of the [WC] class, might be a heterogeneous group, with many representatives actually being H-normal CSs (e.g. NGC6629, NGC6891, Mendez et al. 1988; and NGC6543, Mendez et al. 1990). It would be useful to have a better picture of the abundances of these stars, since indeed some of them might be hybrid between the extreme H-deficiency encountered in [WC] CSs and the H-normal CSs.

${ }^{7}$ Based on the relative numbers of known PG1159 CSs compared to [WC] CSs (Jeffery et al. 1996), and on the [WC] CS statistics of Tylenda (1996). 
As for the origin of [WC] CSs, after two decades of progress in obtaining model Asymptotic Giant Branch (AGB) stars with the appropriate abundances, recent Infrared Space Observatory (ISO) observations have revealed a large deficiency in our understanding. Before 1997, it appeared that the only evolutionary scenario able to eliminate hydrogen from the envelope of an AGB star was the born-again scenario (e.g. Iben et al. 1983). According to this scenario, an evolved CS already on the white dwarf (WD) cooling track can experience a last helium shell flash, briefly return to the AGB, and then evolve once again along the CS horizontal track toward the WD domain. This scenario, observationally confirmed by several objects (V605 Aql, Clayton \& De Marco 1997; Sakurai's object (V4334 Sgr), Duerbeck \& Benetti 1996; FG Sagittae, Gonzalez et al. 1998; A30 and A78, Jacoby \& Ford 1983), is now thought not to be the sole channel for H-deficient CSs.

Since 1997, a series of theoretical papers (Herwig et al. 1997, 1999, Herwig 2000, 2001, Blöcker 2001) showed that H-deficiency, together with carbon and oxygen over-abundances, like those determined for [WC] CSs, could be achieved by two additional scenarios: (i) a late thermal pulse (LTP) during the post AGB evolution, but not as late as the WD cooling track, and (ii) a thermal pulse at the end of the AGB evolution (AGB final thermal pulse or AFTP), when the envelope mass is already low enough that hydrogen can be diluted down. ISO observations of oxygen- as well as carbon-rich dust around [WC] CSs, would imply that almost all the [WC] CSs were oxygen-rich AGB stars till recently, in contrast with model predictions (Herwig et al. 1999). Alternative interpretations are speculative, but it appears now certain that some major piece is still missing from the puzzle of [WC] CSs.

\section{Stellar Wind Abundances of [WC] Central Stars}

The questions, when dealing with the abundances of [WC] CSs (or indeed of any CSs), are: "What do we know?" and "Can we do any better?". The first question has to do with uncertainties. Statistics and uncertainties are at the base of our knowledge, yet they are usually underestimated in size and importance. Abundances are determined with different non-LTE codes (and code versions), which can (e.g. De Marco et al. 2001) produce discrepant results when applied to the same observations. Additionally, the fitting technique, the choice of diagnostic lines and the observations' quality, can all contribute to the size of the uncertainty. The uncertainties quoted by individual papers are, in general, too optimistic, since external uncertainties (code-to-code and code-version differences) are seldom taken into account.

The second question "Can we do any better?", has to do with whether it is worth our while to improve on the old work, for instance, modeling more stars or re-modeling the same stars with more sophisticated codes. Our current knowledge is already good enough to conclude that [WCL] evolve into [WCE] CSs. However, we are still left with fundamental questions as to the origin of the whole class. Therefore only results which can help us discriminate between different evolutionary scenarios are worth pursuing. For instance can we discriminate between AFTP (some hydrogen left) and LTP (almost no hydrogen 
Table 1. Abundances of [WC] CSs. Some objects have been analysed more than once by different authors. The later studies reveal that the $\mathrm{C} / \mathrm{He}$ discrepancy between [WCL] and [WCE] CSs might not be real. Reference codes are listed in the Reference section, except for CRO02 which corresponds to Crowther et al. (in preparation).

\begin{tabular}{|c|c|c|c|c|c|c|c|}
\hline Star & Class & $\overline{\mathrm{H}}$ & $\begin{array}{c}\mathrm{He} \\
\% \mathrm{~b}\end{array}$ & & $\mathrm{O}$ & $\mathrm{N}$ & Ref. \\
\hline K 2-16 & WC11 & $<1$ & 45 & 50 & 5 & 1 & LH98 \\
\hline \multirow{2}{*}{ He $3-1333$} & WC11,WC10 & $\leq 0.5$ & 42 & 50 & 8 & $<1$ & LHJ96 \\
\hline & & 0 & 34 & 52 & 14 & - & DC98 \\
\hline \multirow[t]{2}{*}{ M 4-18 } & WC11,WC10 & $\leq 10$ & 46 & 50 & 4 & $<0.5$ & LHJ96 \\
\hline & & $\leq 4$ & 31 & 56 & 13 & - & DC99 \\
\hline \multirow[t]{2}{*}{ He $2-113$} & WC11,WC10 & $\leq 7$ & 44 & 50 & 6 & 0.5 & LHJ96 \\
\hline & & 0 & 34 & 54 & 13 & - & DC98 \\
\hline PM 1-188 & WC11,WC10 & 1 & 42 & 50 & 7 & 1 & LH98 \\
\hline \multirow[t]{2}{*}{ SwSt 1} & WC9 & $\leq 10$ & 44 & 53 & 3 & - & LH98 \\
\hline & & $\overline{\leq} 10$ & 53 & 32 & 15 & - & DEM02 \\
\hline He $2-459$ & WC9 & $\leq 2$ & 40 & 50 & 10 & - & LH98 \\
\hline He 2-99 & WC9 & $\leq 2$ & 50 & 40 & 10 & - & LHJ96 \\
\hline \multirow[t]{2}{*}{$\mathrm{BD}+30^{\circ} 3639$} & WC9 & $\leq 7$ & 45 & 50 & 5 & - & LHJ96 \\
\hline & & - & 55 & 35 & 10 & - & CRO02 \\
\hline \multirow[t]{2}{*}{ NGC 40} & WC8 & $\leq 2$ & 40 & 50 & 10 & - & LHJ96 \\
\hline & & 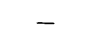 & 52 & 37 & 10 & - & CRO02 \\
\hline He $2-43$ & WC & $\leq 2$ & 40 & 50 & 10 & - & LH98 \\
\hline NGC 6369 & WC4 & - & 56 & 30 & 14 & - & KH97b \\
\hline \multirow[t]{2}{*}{ NGC 6751} & WC4 & - & 36 & 12 & 5 & - & KH97a \\
\hline & & - & 47 & 45 & 10 & - & CRO02 \\
\hline IC 1747 & WC,WC4 & - & 69 & 25 & 6 & $0:$ & KH97b \\
\hline NGC 1501 & WC4,WO4 & - & 50 & 35 & 15 & 0 : & KH97b \\
\hline NGC 7026 & WC,WO3 & - & 70 & 20 & 10 & $0:$ & KH97b \\
\hline Sand 3 & WC2-3,WO1 & - & 54 & 31 & 15 & - & KH97a \\
\hline NGC 5189 & WC2,OVI,WO1 & - & 77.7 & 16 & 8 & 0.3 & KH97b \\
\hline NGC 2452 & WC3,OVI & - & 78 & 15 & 7 & $0:$ & KH97b \\
\hline PB 6 & WC3?,WO1 & - & 61.7 & 24 & 14 & 0.3 & KH97b \\
\hline \multirow[t]{2}{*}{ NGC 6905} & WC3,WO1 & - & 60 & 25 & 15 & 0 & KH97b \\
\hline & & - & 49 & 41 & 10 & - & CRO02 \\
\hline NGC 2867 & WC3 & - & 66 & 25 & 9 & $0:$ & KH97b \\
\hline He 2-55 & WC3 & - & 79 & 15 & 6 & $0:$ & KH97b \\
\hline
\end{tabular}




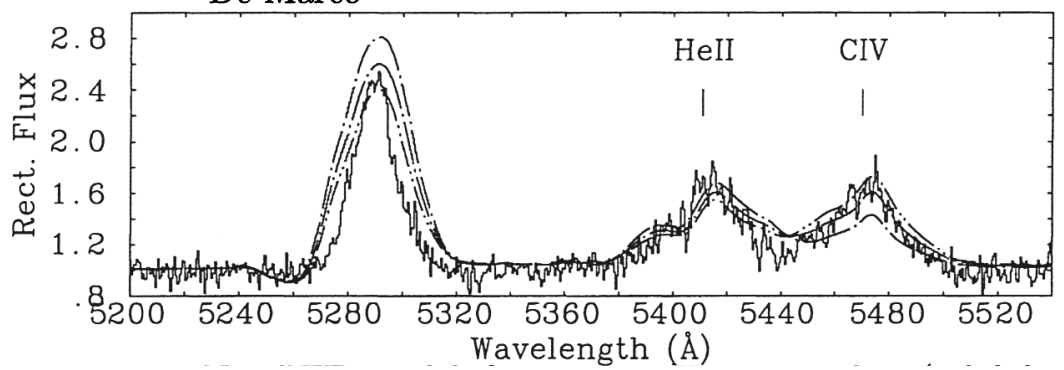

Figure 1. Non-LTE model fits to the [WO1] Sand 3 (solid line; Crowther et al., this meeting). Dashed line: $\mathrm{X}(\mathrm{C})=55 \%$; dotted line: $\mathrm{X}(\mathrm{C})=41 \%$; dot-dashed line: $\mathrm{X}(\mathrm{C})=25 \%$. Reasonably large abundance differences correspond to similar fits.

left) by determining better hydrogen abundances? Can we measure overshoot efficiency by determining accurate oxygen abundances?

For carbon and helium, the most abundant elements, abundances can be determined with reasonable accuracy. Many carbon lines exist for all of its different ionization stages (no He I lines exist for stars earlier than [WC7] [Crowther et al. 1998]). Abundance uncertainties of two stars obtained with the same code, overall if the two stars are in similar ionization stages, can be as low as $10-15 \%$. We call these relative uncertainties. Different codes or different code versions will bring that uncertainty up to about $40 \%$ (see Fig. 1). We call these absolute uncertainties. The $\mathrm{C} / \mathrm{He}$ ratio discrepancy between [WCL] and [WCE] (De Marco \& Barlow 2001) appears, in light of recent results (Crowther et al., this meeting; Table 1), to be due to code and code-version differences, and can now be dismissed. Further determinations of the abundances of these elements might not bring a substantial change to the picture of [WC] CSs.

The hydrogen abundance estimates in the literature vary from no hydrogen to $\sim 10 \%$ by mass (Table 1 ). The amount of hydrogen left in the atmosphere of a [WC] CSs would be paramount in understanding its evolution. It would also be important for understanding the presence of hydrogenated dust, such as PAHs, which is thought to form in the AGB/post-AGB outflow. For instance the interpretation of Waters et al. (1998) of the nature of the PAHs and silicate dust around the [WC9] CS BD $+30^{\circ} 3639$, was based around the estimate that some [WCL] have leftover hydrogen (Leuenhagen et al. 1996). However, as it was shown by De Marco, Barlow \& Storey (1997), contamination of the stellar spectrum by nebular material with unusually high outflow velocity, can lead to an over-estimate of the stellar hydrogen abundance. The determination of the hydrogen abundance could therefore be attempted, with high resolution spectroscopy (R 50000) and a full analysis of the PN and star, but the returns on the time spent cannot be guaranteed.

The abundance of nitrogen in the envelope of [WC] CSs is very low. Indeed we do not expect to observe nitrogen if the CS has lost its entire envelope and its H-rich burning shell. The fact that we do, could be a tracer of leftover hydrogen, or could be due to hot bottom-burning conversion of carbon to nitrogen during the AGB phase of the more massive CSs (those that appear at the center of Type I PNe; Kingsburgh \& Barlow 1994). Its abundance (Table 1) can be considered 

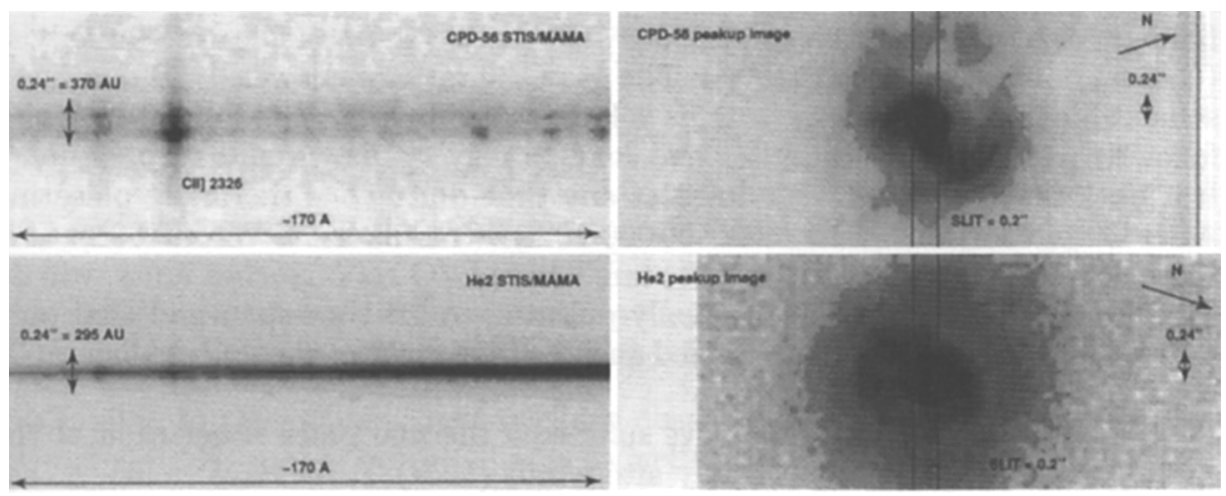

Figure 2. STIS spectra (left) and long-pass images (right) of the [WC10] CSs CPD-56 8032 (top) and He2-113 (bottom).

accurate to $30 \%$ (relative), or $\sim 60 \%$ (absolute). A systematic study of nitrogen abundances in [WC] CSs could therefore be fruitful.

Knowing the oxygen abundance to a couple of percent would provide a check of the dredge-up predicted by the models (e.g. Herwig et al. 1999). Unfortunately very few and weak oxygen lines ([WCL] CSs) and the susceptibility of the oxygen spectrum to the exact ionization structure ([WCE] CSs), means that the relative oxygen abundance uncertainty is $\sim 30 \%$, while the absolute one is $\sim 100 \%$. Further analysis might therefore not improve our overall knowledge. Last, it should be pointed out there might be evidence of a below-solar iron abundance for the H-deficient CSs (NGC40, Crowther et al., priv. comm.; PG1159 stars, Werner, this meeting). Iron under-abundance in CSs, whose precursor should have inherited a near-solar abundance, might be an indication of s-processing during the AGB. The abundance of iron can be determined for [WC] CSs by carefully fitting of the UV pseudo-continuum. This line of investigation is worth pursuing, as it does constrain the amount of $s$-processing during the AGB evolution. This is of general importance since AGB $s$-processing is not fully understood.

\section{The C/O Ratio, from Star to Dust}

Almost all [WCL] CSs exhibit carbon (PAHs) as well as oxygen (amorphous and/or crystalline silicates) dust chemistry (e.g. Szczerba et al. 2001; Cohen 2001 ; the only [WCL] CSs that does not, is NGC 40 [Hony et al. these proceedings]). Two [WCL] CSs have been analyzed in detail: CPD-56 ${ }^{\circ} 8032$ ([WC10]; Cohen et al. 1999) and $\mathrm{BD}+30^{\circ} 3639$ ([WC9]; Waters et al. 1998). Their dust is hot, and hence, must be near the star. For both objects these authors deduce an age for the O-rich dust of $\sim 1000 \mathrm{yr}$.

The simplest explanation of these observations is that the dust might be tracing the O-rich and later C-rich phases of the progenitor AGB star, implying that the [WCL] CSs with double dust chemistry have been on the AGB very 
recently and that until then, they were all O-rich. If the AGB mass-loss outflow has $\mathrm{C} / \mathrm{O}<1$, then the condensing dust will be O-rich (silicates), otherwise $(\mathrm{C} / \mathrm{O}>1)$, it will be $\mathrm{C}$-rich $(\mathrm{PAH})$. The gas $\mathrm{C} / \mathrm{O}$ ratio of a lower-AGB star has a solar value $(\sim 0.4$, by number). This is later raised by the third dredge-up which follows the AGB thermal pulses and may or may not reach and surpass unity. If it does reach unity, it may do so at any time during the thermally-pulsating AGB life of the star, which lasts $500000-10^{6} \mathrm{yr}$ (Vassiliadis \& Wood 1994). It is therefore expected that those AGB stars whose $\mathrm{C} / \mathrm{O}$ ratio reaches unity, will do so at some time during the thermally-pulsating AGB time-span and that only a minority of them will have reached such a stage right at the end of their AGB life.

[WC] CSs are expected to have suffered a thermal pulse either right at the end of the AGB (AFTP) or shortly afterwords (LTP). It is therefore likely, that they were changed into [WCL] CSs recently. However, it is strange that at the time of such pulse the precursor of all [WCL] CSs was O-rich. On average we would in fact expect the distributions of C/O ratios in AGB stars that do become [WC] CSs to be the same as for objects that will remain H-rich. There is no reason why an AFTP or LTP should operate preferentially in those AGB stars which suffered limited or inefficient third dredge-up.

To determine the history of the [WC] CSs we obtained UV spectra of three [WCL] CSs in order to measure their nebular C/O ratios as a function of nebular radius. PN material at different distances from the CS should be tracing the AGB mass-loss history, and therefore have an enrichment characteristic of the third dredge-up efficiency. Of the three [WCL] CSs, CPD- $56^{\circ} 8032$ and He2113 have very high $\mathrm{PN} \mathrm{C} / \mathrm{O}$ ratios (Table 2) and strong PAH features. On the other hand, SwSt1 has $\mathrm{C} / \mathrm{O}<1$, and weak PAH features. The $\mathrm{C} / \mathrm{O}$ ratios were determined from the $\mathrm{C}$ II line $\lambda 2326$ and the $\mathrm{O}$ II lines $\lambda 2470$ (SwSt1) and $\lambda \lambda 3727,7325$ (CPD-56 8032 and He2-113). The reddening was determined self-consistently using the $\mathrm{O}$ II $\lambda 2470-\lambda 7325$ baseline (SwSt1) or it was kept constant at the values determined by De Marco et al. (1997; CPD-56 ${ }^{\circ} 8032$ and $\mathrm{He} 2-113)$. The stellar contribution to the $\mathrm{C}$ II line $\lambda 2326$ was subtracted using model predictions (De Marco \& Crowther 1998, De Marco et al. 2001). From this it was determined that SwSt1's AGB precursor cannot have suffered substantial third dredge-up (its $\mathrm{C} / \mathrm{O}$ ratio is close to solar throughout the PN, possibly increasing only in the recent past, near the star) so that it might have been an O-rich AGB star till the very end, when an LTP/AFTP converted it to a [WC] CS. On the other hand, the precursor of CPD- $56^{\circ} 8032$ must have been losing mass with an already high $\mathrm{C} / \mathrm{O}$ ratio since its value is high throughout the nebula (for He2-113, unfortunately, we do not have estimates far from the star). This star, must have therefore been C-rich $(\mathrm{C} / \mathrm{O}>1)$ for at least a few thermal pulses, excluding that it was an O-rich AGB star only $1000 \mathrm{yr}$ ago, as suggested by the temperature of its hot O-rich dust. which could not AGB star.

We therefore deduce that the double dust around at least some of these [WCL] CSs must remain around the star for a longer time than the $\sim 1000 \mathrm{yr}$ determined by Cohen et al. (1999). One suggestion is that the dust is stored in a disk (Cohen et al. 2002). This idea is supported by the observation that, in the case of CPD- $56^{\circ} 8032$, there appear to be a 5 -yr periodic dimming of the light by $\sim 1$ mag. Although the periodicity needs to be confirmed (since it could 
Table 2. Parameters and dust properties of three [WCL] central stars.

\begin{tabular}{lllllll}
\hline Object & $\begin{array}{l}\text { CSPN } \\
\text { Class }\end{array}$ & $\begin{array}{l}\mathrm{T}_{*} \\
(\mathrm{kK})\end{array}$ & $\begin{array}{l}\mathrm{C} / \mathrm{O} \\
\text { Star }\end{array}$ & $\begin{array}{l}\text { C/O } \\
\text { PN }\end{array}$ & PAHs & Silicates \\
\hline SwSt1 & [WC9p] & 43.0 & 2.6 & 0.71 & Weak & Strong \\
CPD-56 & [WC10] & 34.5 & 5.0 & 9.8 & Strong & Strong \\
He2-113 & [WC10] & 31.2 & 5.5 & 7.5 & Strong & Strong \\
\hline \hline
\end{tabular}

be similar to the random light declines observed for $\mathrm{R}$ Coronae Borealis stars (Clayton 1996) and other [WC] CSs as well as massive WC stars), it could be caused by a disk precessing with a 10 year period (Cohen et al. 2002). This could be the disk around a binary system, or around a binary companion. In the latter case, the companion, with a $\sim 5$ yr period, would be dragging with itself the dusty disk. In Fig. 2 we present the HST observations of CPD-56 $6^{\circ} 8032$ and He2-113. Compared to the spectrum of He2-113 (lower left panel), the spectrum of CPD- $56^{\circ} 8032$ (upper left panel) shows what looks like two spectra. The possibility that they belong to two individual stars was excluded as well as the possibility that it might be due to an instrumental problem. The spectrum of CPD-56 8032 was taken on April 1-5, 2001, during the last observed lightcurve decline. We therefore presume that we are observing the dust puff or disk obscuring the direct starlight. What we see is the light reflected from dust which is above and below the star. Further conclusions await a more complete analysis of the data (De Marco et al., in preparation).

\section{Conclusions}

1) Through non-LTE modeling of [WC] CSs, it is now established that they form a homogeneous evolutionary sequence. Recent results with the latest codes have determined that there is no abundance discrepancy between the cooler [WCL] and hotter [WCE] CSs. Additionally, the classification gap which sees most of the [WC] CSs grouped in either spectral types [WC11]-[WC8] or [WC6][WO1], should not be interpreted on the grounds of evolutionary speed. It is rather generated by a sensitivity of the strength of the $\mathrm{C}$ III $\lambda 5696$ diagnostic line at certain temperature and mass-loss values (Crowther et al. 2002).

2) The uncertainties on the abundance determinations are larger than one is prone to think. Hydrogen in particular is extremely hard to detect because of contamination from the strong PN line and from the He II line for the hotter [WC] CSs. Carbon and helium are the best elements to determine abundances for, with relative uncertainties as low as $15 \%$. Nitrogen and oxygen would be very important elements to measure accurately, but the weakness and paucity of spectral lines make their abundance affected by substantial uncertainty.

3) The double dust chemistry problem, affecting almost all of the known [WCL] CSs is in serious contrast with single star evolutionary models. Alternative scenarios include a dust disk around a companion or a binary system. 
Acknowledgments. Thanks to Slawomir Gorny for help in understanding the WELS and George Jacoby for helpful comments. All of my gratefulness and affection go to Janet Asimov for allowing this research to happen.

\section{References}

Blöcker, T. 2001, Ap\&SS, 275, 1

Clayton, G. C. 1996, PASP, 108, 225

Clayton, G. C., \& De Marco, O. 1997, AJ, 114, 2679

Cohen, M., et al. 1999, ApJ, 513, L 135

Cohen, M. 2001, Ap\&SS, 275, 103

Cohen, M., Barlow, M. J., Liu, X.-W., Jones, A. F. 2002, MNRAS, in press

Crowther, P. A., De Marco, O., \& Barlow, M. J. 1998, MNRAS, 296, 367

Crowther, et al. 2002, A\&A, in press

De Marco, O., Barlow, M. J., \& Storey, P. J. 1997, MNRAS, 292, 86

De Marco, O., \& Crowther, P. A. 1998, MNRAS, 296, 419 (DC98)

De Marco, O., \& Crowther, P. A. 1999, MNRAS, 306, 931 (DC99)

De Marco, O., et al. 2001, MNRAS, 328, 527 (DEM02)

De Marco, O., \& Barlow, M. J. 2001, ApSS, 275, 53

Duerbeck, H. W., \& Benetti, S. 1996, ApJ, 468, L 111

Gonzalez, G., et al. 1998, ApJS, 114, 133

Herwig, F., et al. 1999, A\&A, 350, 89

Herwig, F., et al. 1997, A\&A, 324, L81

Herwig, F. 2000, A\&A, 360, 952

Herwig, F. 2001, Ap\&SS, 275, 15

Hillier, D. J., \& Miller, D. L. 1998, ApJ, 496, 407

Iben, I., et al. 1983, ApJ, 264, 605

Jacoby G. H., \& Ford, H. C. 1983, ApJ, 266, 298

Kingsburgh, R. L., \& Barlow, M. J. 1994 MNRAS, 271, 257

Koesterke, L., \& Hamann, W.-R. 1997a, A\&A, 320, 91 (KH97a)

Koesterke, L., \& Hamann, W.-R. 1997b, in IAU 180, Planetary Nebulae, eds. H.J. Habing \& H.J.G.L.M., Kluwer, 114 (KH97b)

Leuenhagen,U., Hamann, W.-R., \& Jeffery, C.S. 1996, A\&A, 320, 91 (LHJ96)

Leuenhagen,U., \& Hamann, W.-R. 1998, A\&A, 330, 265 (LH98)

Mendez, R.H., Herrero,A., \& Manchado, A. 1990, A\&A, 229, 152

Mendez, R.H., et al. 1988, A\&A, 190, 113

Szczerba, R., et al. 2001, ApSS, 275, 113

Tylenda, R., 1996, in ASP Conf. Ser. Vol. 96, Hydrogen-Deficient Stars, ed. C. S. Jeffery \& U. Heber (San Francisco; ASP), 101

Tylenda, R., Acker, A., \& Stenholm, B. 1993, A\&AS, 102, 595

van der Hucht, K. A., et al. 1981, Sp.Sci.Rev., 28, 227

Vassiliadis, E., \& Wood, P.R. 1994, ApJS, 92, 125

Waters, L. B. F. M. et al., 1998, A\&A, 331, L 61

Werner, K. 2001 Ap\&SS, 275, 27 